\title{
SOME PROPERTIES OF $p(n)$ AND $c(n)$ MODULO POWERS OF 13
}

\author{
BY \\ A. O. L. ATKIN AND J. N. O'BRIEN
}

\section{Introduction. Let}

$$
\begin{aligned}
x & =e^{2 \pi i \tau}, \quad \operatorname{Im} \tau>0, \quad|x|<1, \\
f(x) & =\prod_{r=1}^{\infty}\left(1-x^{r}\right), \\
\eta(\tau) & =e^{\pi i \tau / 12} f(x) \\
1 / f(x) & =\sum_{n=0}^{\infty} p(n) x^{n}, \\
j(\tau) & =\sum_{n=-1}^{\infty} c(n) x^{n}=\left(1+240 \sum_{n=1}^{\infty} \sigma_{3}(n) x^{n}\right)^{3} / x f^{24}(x)-744,
\end{aligned}
$$

where

$$
\sigma_{3}(n)=\sum_{d \mid n} d^{3} .
$$

Then $p(n)$ is just the number of unrestricted partitions of $n$, and $c(n)$ is the Fourier coefficient of Klein's modular invariant. For primes $q \leqq 11$, there exist congruence properties of the type first found by Ramanujan. In fact

(1) if $n \equiv 0\left(\bmod 2^{a} 3^{b} 5^{c} 7^{d} 11^{e}\right)$, then $c(n) \equiv 0\left(\bmod 2^{3 a+8} 3^{2 b+3} 5^{c+1} 7^{d} 11^{e}\right)$,

and

(2) if $24 n \equiv 1\left(\bmod 5^{c} 7^{d} 11^{e}\right)$, then $p(n) \equiv 0\left(\bmod 5^{c} 7^{[(d+2) / 2]} 11^{e}\right)$.

These are proved in Watson [2], Lehner [3], [4], Atkin [5]. For primes $q>11$ this type $\left({ }^{1}\right)$ of congruence does not persist.

Newman [6], in the context of $p(n)$, has proposed the question:

Given $a, m$, is $p(n) \equiv a(\bmod m)$ soluble for an infinity of $n$ ?

We may ask further:

(4) Given $a, m$, is $p(n) \equiv a(\bmod m)$ soluble for values of $n$ with positive density?

The best hope of establishing (3) seems to be that one may exhibit explicit congruences of the form

$$
p(h(n)) \equiv a(\bmod m)
$$

Received by the editors May 3, 1966.

(1) I.e., $p(k n+l) \equiv 0(\bmod m)$ where no primes divide $k$ which do not also divide $m$. 
and in particular if $h(n)=b n+c$ is a linear function of $n$, we have (4) also. The same questions, of course, arise for $c(n)$, and indeed for the Fourier coefficients of other suitable modular forms and functions. In this paper we make some contribution to the solution of these problems when $m$ is a power of 13. We confine ourselves to $p(n)$ and $c(n)$, both for simplicity and because these coefficients have been most extensively studied, but the reader will see that the methods are applicable to the Fourier coefficients of any negative power of $\eta(\tau)$ or any entire function on $\Gamma_{0}(13)$.

1.1. Congruence properties of $c(n)$. Newman [7] shows that

$$
c\left(13^{2} n\right) \equiv 8 c(13 n)(\bmod 13) \text {, }
$$

and, with

$$
\begin{aligned}
& t(n)=-c(13 n) \equiv c(13 n) / c(13)(\bmod 13), \\
& t(n p)-t(n) t(p)+p^{-1} t(n / p) \equiv 0(\bmod 13),
\end{aligned}
$$

where $p \neq 13$ is prime $\left({ }^{2}\right)$.

Now, by using the "accident" that $c(91) \equiv 0(\bmod 13)$ he infers that

$$
\begin{gathered}
c\left(7.13^{\alpha}\right) \equiv 0(\bmod 13) \quad \text { if } \alpha \geqq 1, \\
c(91 n) \equiv 0(\bmod 13) \quad \text { if }(n, 7)=1 .
\end{gathered}
$$

Both these results prove that $c(n) \equiv 0(\bmod 13)$ infinitely often; the second also shows that $c(n) \equiv 0(\bmod 13)$ in positive density. One can now with a little calculation show also that $c(n)$ fills all residue classes $(\bmod 13)$ infinitely often, but 'positive density' seems more intractable. We may regard (7) as expressing a 'multiplicative' property of $c(13 n)(\bmod 13)$, which takes the form $(6)$ in the ramified case $p=13$. Indeed Newman's proof is based on the fact that $c(13 n) \equiv-\tau(n)(\bmod 13)$, where $\tau(n)$ is defined by

$$
\sum_{n=1}^{\infty} \tau(n) x^{n}=x f^{24}(x)
$$

and satisfies

$$
\tau(n p)-\tau(n) \tau(p)+p^{11} \tau(n / p)=0,
$$

as found by Ramanujan [10] and proved by Mordell [11]. In this paper we prove a generalization of (6):

THEOREM 1. For all $\alpha \geqq 1$ there exists a constant $k_{\alpha}$ not divisible by 13 such that for all $n$

$$
c\left(13^{\alpha+1} n\right) \equiv k_{\alpha} c\left(13^{\alpha} n\right)\left(\bmod 13^{\alpha}\right) .
$$

$\left({ }^{2}\right)$ Here and later we use the reciprocal of $a\left(\bmod 13^{\alpha}\right)$ freely when $(a, 13)=1$. We also write, for any number-theoretic coefficient $t(n), t(n)=0$ if $n$ is nonintegral. 
We have also overwhelming evidence for the following generalization of (7):

CONJECTURE 1 . Let $\alpha \geqq 1$ be integral, and write

$$
t(n) \equiv c\left(13^{\alpha} n\right) / c\left(13^{\alpha}\right)\left(\bmod 13^{\alpha}\right) .
$$

Then if $p \neq 13$ is prime we have

$$
t(n p)-t(n) t(p)+p^{-1} t(n / p) \equiv 0\left(\bmod 13^{\alpha}\right) .
$$

We observe that the final congruence of Conjecture 1 is exactly analogous to the identities in Hecke's theory of modular forms of negative dimension. Thus it seems that, in a sense, Newman's congruences (6) and (7) exist in their own right, although it happens that $c(13 n) \equiv-\tau(n)(\bmod 13)$, and this enables $(7)$ to be proved.

We use Theorem 1 and a good deal of actual computation to establish:

THEOREM 3. $c(n) \equiv 0\left(\bmod 13^{3}\right)$ for infinitely many $n$.

THEOREM 4. For all $\alpha \geqq 1$, and all a with $(a, 13)=1$, there exist infinitely many $n$ such that $c(n) \equiv a\left(\bmod 13^{\alpha}\right)$.

1.2. Congruence properties of $p(n)$. These are more involved than those of $c(n)$, as we might expect from the fact that $\eta(\tau)$ is a modular form of half-integral dimension and complicated multiplier system. In order to obtain some simplification, we define

$$
P(N)=p(n) \quad \text { if } N=24 n-1,
$$

$$
P(N)=0 \quad \text { if } N<-1 \text { or } N \not \equiv-1(\bmod 24) \text { or } N \text { is nonintegral. }
$$

After subsection 1.1, we shall describe the analogous position here more briefly. We have

THEOREM 2. For all $\alpha \geqq 1$ there exists an integral constant $K_{\alpha}$ not divisible by 13 such that for all $N$

$$
P\left(13^{\alpha+2} N\right) \equiv K_{\alpha} P\left(13^{\alpha} N\right)\left(\bmod 13^{\alpha}\right)\left({ }^{3}\right) .
$$

We also conjecture

CONJECTURE 2 . Let $\alpha \geqq 1$, and $p \neq 13$ be a prime $\geqq 5$. Then there exists a constant $k=k(p, \alpha)$ such that for all $N$

$$
P\left(p^{2} \cdot 13^{\alpha} N\right)-\left\{k-\left(-3 \cdot 13^{\alpha} N / p\right) p^{-2}\right\} P\left(13^{\alpha} N\right)+p^{-3} P\left(13^{\alpha} N / p^{2}\right) \equiv 0\left(\bmod 13^{\alpha}\right)\left({ }^{4}\right),
$$

where $(a \mid b)$ is the quadratic reciprocity symbol.

THEOREM 5. Conjecture 2 is valid if $\alpha=1$ or $\alpha=2$.

( $\left.{ }^{3}\right)$ In these forms, of course, the congruences are nugatory unless $N$ is such that $13^{\alpha} N$ $\equiv-1(\bmod 24)$.

( $\left.{ }^{4}\right)$ Since the factor multiplying $P\left(13^{\alpha} N\right)$ cannot be written as $P\left(13^{\alpha} N_{1}\right)$ the use of $t(n)$ as in Conjecture 1 offers no advantage. 
Again, we have a kind of multiplicative property modulo $13^{\alpha}$, and Theorem 2 is essentially the case $p=13$ of Conjecture 2 .

Newman [8], [9] obtains the case $\alpha=1$ of Theorem 2, and (although he does not state it in our form) of Conjecture 2, using Zuckermann's result

where

$$
p(13 n+6) \equiv 11 p_{11}(n)(\bmod 13)
$$

$$
\sum_{n=0}^{\infty} p_{r}(n) x^{n}=f^{r}(x)
$$

and his own results on $p_{r}(n)$ for $r$ odd, $1 \leqq r \leqq 23$, with $r=11$. He now uses the “accident" $P(2015)=p(84) \equiv 0(\bmod 13)$ to establish

$$
\begin{gathered}
P\left(2015 \cdot 13^{2 n}\right) \equiv 0(\bmod 13) \quad(n \geqq 0), \\
P\left(2015 n^{2}\right)=p\left(84 n^{2}-\left(n^{2}-1\right) / 24\right) \equiv 0(\bmod 13) \quad \text { if }(n, 6)=1 .
\end{gathered}
$$

Note that the result analogous to $(9)$ is $(13)$, which does not give $p(n) \equiv 0(\bmod 13)$ in positive density. If we search for an "accident" involving $k(p, \alpha)$ rather than $P\left(13 N_{0}\right)$ we do in fact obtain a linear congruence from Newman's results:

THEOREḾ 6. $P\left(59^{3} \cdot 13 N\right) \equiv 0(\bmod 13)$ if $(N, 59)=1$.

Thus, for example, in terms of $p(n)$, with $N=24.59 n+1$,

$$
p\left(59^{4} \cdot 13 n+111247\right) \equiv 0(\bmod 13) .
$$

Using Theorem 2, we can prove

THEOREM 7. $p(n) \equiv 0\left(\bmod 13^{4}\right)$ for infinitely many $n$.

THEOREM 8. For all $\alpha \geqq 1$, and all a with $(a, 13)=1$, there exist infinitely many $n$ such that $p(n) \equiv a\left(\bmod 13^{\alpha}\right)$.

Finally, using Conjecture 2 in the proved case $\alpha=2$, we obtain

THEOREM 9. $p\left(3373 n^{2}-\left(n^{2}-1\right) / 24\right)=P\left(13^{2} \cdot 479 n^{2}\right) \equiv 0\left(\bmod 13^{2}\right)$ if $(n, 6)=1$.

THEOREM 10. $P\left(97^{2} \cdot 103^{2} \cdot 13^{2} N\right) \equiv 0\left(\bmod 13^{2}\right)$ if $(N / 97)=(N / 103)=-1$.

Thus, for example, in terms of $p(n)$

$$
p(168544110546799 n-6950975499605) \equiv 0\left(\bmod 13^{2}\right) \quad \text { if } n \geqq 1 .
$$

The methods we employ in this paper are essentially elementary, based (as in Watson [2]) on the use of the modular equation between $\eta(169 \tau) / \eta(\tau)$ and $\eta^{2}(169 \tau) / \eta^{2}(13 \tau)$, although modular theory was used in [1] to obtain this equation. Although the forms of some of our results can be obtained easily by deeper methods, the precise number-theoretic details cannot, and we have therefore avoided modular notation as far as possible. The only case where we require modular theory is Lemma 5. We may add that Lehner [4] gives the modular 
equation between $\eta^{2}(13 \tau) / \eta^{2}(\tau)$ and $\eta^{2}(169 \tau) / \eta^{2}(13 \tau)$. This would have sufficed for our results on $c(n)$, but not for those on $p(n)$. We have been occupied with the work described in this paper at various times since 1962, and would like to thank Dr. Morris Newman of the National Bureau of Standards, Washington, D. C., for his constant advice and encouragement.

2.1. Sums of powers of roots of the modular equation. We now write

$$
\begin{aligned}
& \phi(x)=x^{7} f\left(x^{169}\right) / f(x), \\
& g(x)=x f^{2}\left(x^{13}\right) / f^{2}(x) .
\end{aligned}
$$

Then, as shown by O'Brien in [1] the equation connecting $\phi\left(x^{1 / 13}\right)=t$ and $g(x)=g$ may be written

$$
t^{13}+\alpha_{1} t^{12}+\cdots+\alpha_{12} t+\alpha_{13}=0
$$

where

$$
\alpha_{r}=\sum_{\sigma=[(r+2) / 2]}^{7} \beta_{r \sigma} g^{\sigma}
$$

and the $\beta_{r \sigma}$ are integers. We now define $\pi(a)$, for integral $a$, by

$$
13^{\pi(a)} \mid a, \quad 13^{\pi(a)+1} \nmid a .
$$

If $a=b / c$ is rational, we define $\pi(a)=\pi(b)-\pi(c)$, and finally we write $\pi(0)=\infty$ and regard any inequality $\pi(0) \geqq k$ as valid. We clearly have

$$
\begin{gathered}
\pi(a b)=\pi(a)+\pi(b), \\
\pi(a+b) \geqq \min (\pi(a), \pi(b)),
\end{gathered}
$$

with equality unless $\pi(a)=\pi(b)$. With this notation, we have

LEMMA 1. $\pi\left(\beta_{r \sigma}\right) \geqq[(13 \sigma-7 r+13) / 14]$.

The proof consists of direct verification using the calculated values of $\beta_{r \sigma}$ in [1], which we give in Appendix C.

It is possible to deduce some cases of Lemma 1 a priori, as shown in [1], but not all. We now denote by $S_{r}$ the sum of the $r$ th powers of the roots of (17), regarded as an equation in $t$. Evidently we have

$$
S_{r}=\sum_{\rho=1}^{\infty} a_{r \rho} g^{\rho}
$$

where in $\operatorname{fact}\left({ }^{5}\right)$

$$
a_{r \rho}=0 \text { unless }[(7 r+12) / 13] \leqq \rho \leqq 7 r .
$$

We now prove

LEMMA 2. $\pi\left(a_{r \rho}\right) \geqq[(13 \rho-7 r+13) / 14]$.

(5) Here and in our later inductions it is convenient to write finite sums as $\sum_{\rho}=\sum_{\rho=1}^{\infty}$, since the actual limits do not in most cases affect the argument. We do however usually state without proof the exact limits, which the reader can easily verify, in lemmas. 
This is true for $r=1$ and all $\rho$, by Lemma 1. Suppose it has been established for all $\rho$ and all $r<R$. Then, by Newton's formula

$$
\begin{aligned}
-\sum a_{R \rho} g^{\rho}=\sum_{r=1}^{R-1}\left(\sum_{\sigma} a_{r \sigma} g^{\sigma}\right)\left(\sum_{\tau} \beta_{R-r, \tau} g^{\tau}\right)+R \sum_{\rho} \beta_{R \rho} g^{\rho}, \\
\quad\left(\text { where } \beta_{R \rho}=0 \text { for } R \geqq 14, \text { all } \rho\right)
\end{aligned}
$$

so that

$$
-a_{R \rho}=\sum_{r=1}^{R-1} \sum_{\sigma} a_{r \sigma} \beta_{R-r, \rho-\sigma}+R \beta_{R \rho},
$$

whence, by (19) and (20),

where

$$
\left.\pi\left(a_{R \rho}\right) \geqq \min \{[13 \rho-7 R+13) / 14], \psi(\rho, R)\right\},
$$

$$
\psi(\rho, R)=\min _{\sigma \geqq 1,1 \leqq r \leqq R-1}\{[(13 \sigma-7 r+13) / 14]+[(13(\rho-\sigma+1)-7(R-r)) / 14]\} .
$$

Now

$$
[\lambda / 14]+[\mu / 14] \geqq[(\lambda+\mu-13) / 14]
$$

so that

$$
\psi(\rho, R) \geqq \min _{\sigma \geqq 1,1 \leqq r \leqq R-1}\{[(13 \rho-7 R+13) / 14]\}=[(13 \rho-7 R+13) / 14] .
$$

Hence

$$
\pi\left(a_{R \rho}\right) \geqq[(13 \rho-7 R+13) / 14],
$$

and Lemma 2 is proved by induction. We have also

$$
\pi\left(a_{r \rho}\right) \geqq 1 \text {. }
$$

For $\pi\left(\beta_{R_{\rho}}\right) \geqq 1$ unless $R=13, \rho=7$ and in this case (23) has a term $R \beta_{R_{\rho}}=13 \beta_{13,7}$ and $\pi\left(13 \beta_{13.7}\right)=1$. An easy induction now gives (25).

2.2. The operator $U$. We now define, for any power series

$$
F(x)=\sum_{n \geqq N} \lambda(n) x^{n}
$$

the operator $U\left(=U_{13}\right)$ by

$$
U F(x)=\sum_{13 n \geqq N} \lambda(13 n) x^{n} .
$$

Clearly $U$ is linear so that $U\left\{a_{1} F_{1}(x)+a_{2} F_{2}(x)\right\}=a_{1} U F_{1}(x)+a_{2} U F_{2}(x)$. Further we have

$$
U\left\{F_{1}\left(x^{13}\right) \cdot F_{2}(x)\right\}=F_{1}(x) \cdot U F_{2}(x) .
$$

Finally the elementary properties of roots of unity give

Lemma 3. Suppose that $\omega \neq 1, \omega^{13}=1$. Then $13 U F(x)=\sum_{m=0}^{12} F\left(\omega^{m} x^{1 / 13}\right)$. 
If we now regard (17) as an equation in $t$ with $g$ fixed, the roots are clearly

$$
t=\phi\left(\omega^{m} x^{1 / 13}\right), \quad m=0 \text { to } 12 .
$$

Thus, by Lemma 3, we have

$$
U \phi^{r}(x)=13^{-1} S_{r}
$$

Moreover $U g^{k}(x)=U\left\{\phi^{2 k}(x) \cdot x^{-13 k} \cdot f^{2 k}\left(x^{13}\right) \cdot f^{-2 k}\left(x^{169}\right)\right\}=13^{-1} g^{-k}(x) \cdot S_{2 k}$, by (28) and (29), while similarly $U\left\{\phi(x) \cdot g^{k}(x)\right\}=13^{-1} g^{-k}(x) \cdot S_{2 k+1}$. Using the results of $\$ 2.1$ we derive

LEMMA 4. We have, for $k \geqq 1$,

$$
\begin{array}{r}
U g^{k}(x)=\sum_{r=1}^{\infty} c_{k r} g^{r}(x), \\
U\left\{\phi(x) g^{k}(x)\right\}=\sum_{r=1}^{\infty} d_{k r} g^{r}(x),
\end{array}
$$

where the $c_{k r}$ and $d_{k r}$ are integers satisfying

$$
\begin{aligned}
& \pi\left(c_{k r}\right) \geqq[(13 r-k-1) / 14], \\
& \pi\left(d_{k r}\right) \geqq[(13 r-k-8) / 14], \\
& \pi\left(c_{11}\right)=0, \pi\left(d_{11}\right)=0 .
\end{aligned}
$$

Also in fact

$$
\begin{aligned}
& c_{k r}=0 \text { unless }[(k+12) / 13] \leqq r \leqq 13 k, \\
& d_{k r}=0 \text { unless }[(k+19) / 13] \leqq r \leqq 13 k+7 .
\end{aligned}
$$

3. Proof of congruence properties of $c(n)$. In order to obtain a start for our induction we require

LEMMA 5. $\sum_{n=1}^{\infty} c(13 n) x^{n}=-g(x)+13^{2} U^{2} g(x)$.

We prove this in Appendix A. It can be seen by repeated application of Lemma 4, equation (30), that we may write

$$
J_{\alpha}(x)=\sum_{n=1}^{\infty} c\left(13^{\alpha} n\right) x^{n}=\sum_{r} j_{\alpha r} g^{r}(x), \quad(\alpha \geqq 1),
$$

where in fact $r$ runs from 1 to $13^{\alpha+1}$. We have

LEMMA 6.

$$
\begin{array}{ll}
\pi\left(j_{\alpha \gamma}\right) \geqq[(13 r-2) / 14] & (\alpha \geqq 1), \\
\pi\left(j_{\alpha 1}\right)=0 & (\alpha \geqq 1) .
\end{array}
$$


For

$$
U \sum_{n=1}^{\infty} c\left(13^{\alpha} n\right) x^{n}=\sum_{n=1}^{\infty} c\left(13^{\alpha+1} n\right) x^{n}
$$

and hence, by Lemma 4

$$
j_{\alpha+1, r}=\sum_{\rho} j_{\alpha \rho} c_{\rho r}, \quad \pi\left(j_{\alpha+1, r}\right) \geqq \min _{\rho}\left\{\pi\left(j_{\alpha \rho}\right)+[(13 r-\rho-1) / 14]\right\} .
$$

Thus if we assume (36) for some $\alpha$ and all $r$, we have

$$
\pi\left(j_{\alpha+1, r}\right) \geqq \min _{\rho}\{[(13 \rho-2) / 14]+[(13 r-\rho-1) / 14]\} .
$$

Increasing $\rho$ by 2 increases [(13 $\rho-2) / 14]$ by at least 1 , and decreases $[(13 r-\rho-1) / 14]$ by at most 1 . Hence $\left({ }^{6}\right)$ the $\min _{\rho}$ in (39) is attained for $\rho=1$ or $\rho=2$, and in fact for $\rho=1$, since $[11 / 14]=0$ and $[24 / 14]=1$, whence $\pi\left(j_{\alpha+1, r}\right) \geqq[(13 r-2) / 14],(r \geqq 1)$, which is (36) for $\alpha+1$ and all $r$. Next, assuming (37) for $\alpha$, we have

$$
j_{\alpha+1,1}=\sum_{\rho} j_{\alpha \rho} c_{\rho 1} \text {. }
$$

Now $\pi\left(j_{\alpha 1} c_{11}\right)=0$, by hypothesis and (34), and for $\rho \geqq 2$,

$$
\pi\left(j_{\alpha \rho} c_{\rho 1}\right) \geqq[(13 \rho-2) / 14]+\pi\left(c_{\rho 1}\right) \geqq 1 .
$$

Hence, by (20), we have $\pi\left(j_{\alpha+1,1}\right)=0$, which is (37) for $\alpha+1$. Finally, for $\alpha=1$, (37) is immediate from Lemma 5 , and (36) for $r \geqq 2$ is easily established by the foregoing argument (with something to spare). Lemma 6 is thus proved by induction.

We now define

$$
\gamma_{r s}^{\alpha}=j_{\alpha+1, r} j_{\alpha s}-j_{\alpha r} j_{\alpha+1, s} \quad(\alpha \geqq 1 ; r, s \geqq 1),
$$

so that

$$
\gamma_{r r}^{\alpha}=0, \quad \gamma_{r s}^{\alpha}=-\gamma_{s r}^{\alpha} .
$$

LEMMA 7. $\pi\left(\gamma_{r s}^{\alpha}\right) \geqq \alpha+[(13(r+s)-32) / 14]$.

First for $\alpha=1$ we have by Lemma 6

$$
\begin{aligned}
\pi\left(\gamma_{r s}^{1}\right) & \geqq[(13 r-2) / 14]+[(13 s-2) / 14] \\
& \geqq[(13(r+s)-17) / 14] \geqq 1+[(13(r+s)-32) / 14] .
\end{aligned}
$$

We now have, by (38),

$$
\gamma_{r s}^{\alpha+1}=\sum_{\rho} \sum_{\sigma} \gamma_{\rho \sigma}^{\alpha} c_{\rho r} c_{\sigma s} \quad(\alpha \geqq 1)
$$

and by (32),

$$
\pi\left(c_{\rho r} c_{\sigma s}\right) \geqq[(13 r-\rho-1) / 14]+[(13 s-\sigma-1) / 14] \geqq[(13(r+s)-(\rho+\sigma)-15) / 14] .
$$

$\left({ }^{6}\right)$ The argument of this paragraph is constantly used hereafter, and in future we shall often omit the details. 
Thus assuming Lemma 7 for $\alpha$ and all $r, s$, we have

$$
\pi\left(\gamma_{r s}^{\alpha+1}\right) \geqq \min _{\rho, \sigma}\{\alpha+[(13(\rho+\sigma)-32) / 14]+[(13(r+s)-(\rho+\sigma)-15) / 14]\} .
$$

The $\min _{\rho, \sigma}$ is attained for $\rho+\sigma=3$, whence

$$
\pi\left(\gamma_{r s}^{\alpha+1}\right) \geqq \alpha+[(13(r+s)-18) / 14]=\alpha+1+[(13(r+s)-32) / 14],
$$

which is Lemma 7 for $\alpha+1$ and all $r$, s. Lemma 7 is thus proved by induction. All we actually require later is

$$
\pi\left(\gamma_{r 1}^{\alpha}\right) \geqq \alpha,
$$

but it is not possible to prove (43) alone by induction.

Let now $k_{\alpha}$ not divisible by 13 be chosen so that

$$
j_{\alpha+1,1} \equiv k_{\alpha} j_{\alpha 1}\left(\bmod 13^{\alpha}\right) \text {, }
$$

which is possible by (37) of Lemma 6. Since for all $r$ we have from (43)

$$
j_{\alpha+1, r} j_{\alpha 1}-j_{\alpha r} j_{\alpha+1,1} \equiv 0\left(\bmod 13^{\alpha}\right)
$$

it follows that for all $r$

$$
j_{\alpha+1, r} \equiv k_{\alpha} j_{\alpha r}\left(\bmod 13^{\alpha}\right)
$$

Hence by (35)

$$
J_{a+1}(x) \equiv k_{\alpha} J_{\alpha}(x)\left(\bmod 13^{\alpha}\right),
$$

and equating coefficients of like powers of $x$ we obtain Theorem 1. We may add that Theorem 1 is best possible in the sense that we cannot replace $\left(\bmod 13^{\alpha}\right)$ by $\left(\bmod 13^{\alpha+1}\right)$, which follows from

$$
\pi\left(\gamma_{21}^{\alpha}\right)=\alpha .
$$

The proof of (44) is similar to that of (37), if we write (42) in the form

$$
\gamma_{r s}^{\alpha+1}=\sum_{\rho>\sigma \geqq 1} \sum_{\rho \sigma}^{\alpha}\left(c_{\rho r} c_{\sigma s}-c_{\rho s} c_{\sigma r}\right)
$$

and compute

$$
\pi\left(c_{11} c_{22}-c_{12} c_{21}\right)=1, \quad \pi\left(c_{12}\right)=1 .
$$

We observe next that $c\left(5299 \cdot 13^{3}\right) \equiv 0\left(\bmod 13^{3}\right)$, and hence by Theorem 1

$$
c\left(5299 \cdot 13^{m+3}\right) \equiv 0\left(\bmod 13^{3}\right) \quad(m \geqq 0),
$$

which implies Theorem 3 . To prove Theorem 4 we note that $k_{\alpha} \equiv k_{2}\left(\bmod 13^{2}\right)$ for $\alpha \geqq 2$, while $k_{2}=138 \equiv 41^{3}\left(\bmod 13^{2}\right)$ and 41 is a primitive root of $13^{2}$. Thus $k_{\alpha}$ generates a group of index 3 in the multiplicative group of residues prime to $13^{\alpha}$, and hence by Theorem 1 , if $c\left(13^{\alpha} n_{0}\right)$ equals any residue in one of the cosets, then 
the numbers $c\left(13^{\alpha+m} n_{0}\right)$ for $m \geqq 0$ fill all residues in that coset infinitely often. But $c\left(13^{\alpha} n_{0}\right)$ belongs to the same coset at $c\left(13 n_{0}\right)$, and

$$
c\left(13 n_{0}\right) \equiv-1,-2,6(\bmod 13) \quad \text { for } n_{0}=1,2,5 .
$$

This implies Theorem 4.

4. Proof of Theorems 2, 7, and 8. We now write, for $\alpha \geqq 1$,

$$
\begin{aligned}
L_{2 \alpha-1}(x) & =f\left(\dot{x}^{13}\right) \cdot \sum_{n=1}^{\infty} P\left(13^{2 \alpha-1}(24 n-13)\right) \cdot x^{n} \\
L_{2 \alpha}(x) & =f(x) \cdot \sum_{n=1}^{\infty} P\left(13^{2 \alpha}(24 n-1)\right) \cdot x^{n}
\end{aligned}
$$

LEMMa 8.

$$
\begin{aligned}
L_{1}(x) & =U \phi(x), & & \\
L_{2 \alpha}(x) & =U L_{2 \alpha-1}(x) & & (\alpha \geqq 1), \\
L_{2 \alpha+1}(x) & =U\left(\phi(x) \cdot L_{2 \alpha}(x)\right) & & (\alpha \geqq 1) .
\end{aligned}
$$

We have for (47),

$$
U \phi(x)=f\left(x^{13}\right) \cdot U \sum_{n=1}^{\infty} p(n) x^{n+7}=f\left(x^{13}\right) \cdot \sum_{n=1}^{\infty} p(13 n-7) \cdot x^{n}=L_{1}(x)
$$

and (48) is immediate. Also

$$
\begin{aligned}
U\left(\phi(x) \cdot L_{2 \alpha}(x)\right) & =f\left(x^{13}\right) \cdot U \sum_{n=1}^{\infty} P\left(13^{2 \alpha}(24 n-1)\right) x^{n+7} \\
& =f\left(x^{13}\right) \cdot \sum_{n=1}^{\infty} P\left(13^{2 \alpha}(24(13 n-7)-1)\right) x^{n}=L_{2 \alpha+1}(x) .
\end{aligned}
$$

It now follows by repeated application of Lemma 4 that we may write, for $\alpha \geqq 1$,

$$
\begin{aligned}
L_{2 \alpha-1}(x) & =\sum_{r} k_{\alpha r} g^{r}(x), \\
L_{2 \alpha}(x) & =\sum_{r} l_{\alpha r} g^{r}(x),
\end{aligned}
$$

where

$$
\begin{aligned}
l_{\alpha r} & =\sum_{\rho} k_{\alpha \rho} c_{\rho r} \\
k_{\alpha+1, r} & =\sum_{\rho} l_{\alpha \rho} d_{\rho r} .
\end{aligned}
$$

\section{LEMMA 9.}

$$
\begin{array}{ll}
\pi\left(k_{\alpha r}\right) \geqq[(13 r-9) / 14] & (\alpha \geqq 1), \\
\pi\left(l_{\alpha r}\right) \geqq[(13 r-2) / 14] & (\alpha \geqq 1), \\
\pi\left(k_{\alpha 1}\right)=\pi\left(l_{\alpha 1}\right)=0 & (\alpha \geqq 1) .
\end{array}
$$


First we have by (29)

and

$$
\sum k_{1 r} g^{r}(x)=U \phi(x)=13^{-1} S_{1}=13^{-1} \sum a_{1 r} g^{r}(x)
$$

$$
\pi\left(13^{-1} a_{1 r}\right) \geqq-1+[(13 r+6) / 14] \geqq[(13 r-9) / 14]
$$

by Lemma 2. Next, assuming (54) for $\alpha$ and all $r$ we have by (52) and (32)

$$
\pi\left(l_{\alpha r}\right) \geqq \min _{\rho}\{[(13 \rho-9) / 14]+[(13 r-\rho-1) / 14]\},
$$

and the $\min _{\rho}$ is attained for $\rho=1$, giving (55) for $\alpha$ and all $r$. Finally assuming (55) for $\alpha$ and all $r$, we have by (53) and (33)

$$
\pi\left(k_{\alpha+1, r}\right) \geqq \min _{\rho}\{[(13 \rho-2) / 14]+[(13 r-\rho-8) / 14]\},
$$

and again the $\min _{\rho}$ is attained for $\rho=1$, giving (54) for $\alpha+1$ and all $r$. Thus (54) and (55) are proved for all $\alpha \geqq 1$ by induction. The proof of (56) is analogous to that of (37), if we observe that

$$
\pi\left(c_{11}\right)=\pi\left(d_{11}\right)=\pi\left(k_{11}\right)=0 .
$$

We now write

$$
\begin{aligned}
& \delta_{r s}^{\alpha}=k_{\alpha+1, r} k_{\alpha s}-k_{\alpha r} k_{\alpha+1, s}, \\
& \varepsilon_{r s}^{\alpha}=l_{\alpha+1, r} l_{\alpha s}-l_{\alpha r} l_{\alpha+1, s}, \quad(\alpha \geqq 1 ; r, s \geqq 1),
\end{aligned}
$$

so that

$$
\delta_{r r}^{\alpha}=\varepsilon_{r r}^{\alpha}=0, \quad \delta_{r s}^{\alpha}=-\delta_{s r}^{\alpha}, \quad \varepsilon_{r s}^{\alpha}=-\varepsilon_{s r}^{\alpha}
$$

and

$$
\begin{aligned}
\varepsilon_{r s}^{\alpha} & =\sum_{\rho} \sum_{\sigma} \delta_{\rho \sigma}^{\alpha} c_{\rho r} c_{\sigma s}, \\
\delta_{r s}^{\alpha+1} & =\sum_{\rho} \sum_{\sigma} \varepsilon_{\rho \sigma}^{\alpha} d_{\rho r} d_{\sigma s}
\end{aligned}
$$

LEMMA 10.

$$
\begin{aligned}
& \pi\left(\delta_{r s}^{\alpha}\right) \geqq 2 \alpha-1+[(13(r+s)-46) / 14]+\theta(r, s), \\
& \pi\left(\varepsilon_{r s}^{\alpha}\right) \geqq 2 \alpha+[(13(r+s)-33) / 14],
\end{aligned}
$$

where $\theta(r, s)=1$ if $r+s=3$, and 0 otherwise.

First we have by (54)

$$
\pi\left(\delta_{r s}^{1}\right) \geqq[(13 r-9) / 14]+[(13 s-9) / 14] \geqq 1+[(13(r+s)-44) / 14],
$$

while if $r+s=3$ we have $\pi\left(\delta_{r s}^{1}\right) \geqq 0+1=1$, which proves (61) for $\alpha=1$. Next, assuming (61) for $\alpha$ and all $r$, we have by (59) and (32)

$$
\pi\left(\varepsilon_{r s}^{\alpha}\right) \geqq 2 \alpha-1+\min _{\rho, \sigma}\{[(13(\rho+\sigma)-46) / 14]+\theta(\rho, \sigma)+[(13(r+s)-(\rho+\sigma)-15) / 14]\},
$$


and the $\min _{\rho, \sigma}$ is attained for $\rho+\sigma=3,4$, or 5 , and in fact for $\rho+\sigma=4$, giving (62) for $\alpha$ and all $r, s$. Finally, assuming (62) for $\alpha$ and all $r, s$, we have by (60) and (33)

$$
\pi\left(\varepsilon_{r s}^{\alpha+1}\right) \geqq 2 \alpha+\min _{\rho, \sigma}\{[(13(\rho+\sigma)-33) / 14]+\psi(\rho, \sigma)\},
$$

where

$$
\begin{aligned}
\pi\left(d_{\rho r} d_{\sigma s}\right) \geqq \psi(\rho, \sigma) & =1 \text { if } r+s=\rho+\sigma=3 \\
& =[(13(r+s)-(\rho+\sigma)-29) / 14] \text { otherwise. }
\end{aligned}
$$

The $\min _{\rho, \sigma}$ in (63) is attained for $\rho+\sigma=3$ or 4 , and in fact for $\rho+\sigma=3$, when $r+s>3$, which gives (61) for $\alpha+1$ and all $r, s$; while if $r+s=3$ the $\min _{\rho, \sigma}$ in (63) is attained for $\rho+\sigma=3,4$, or 5 , and in fact for $\rho+\sigma=3$ and 4 , giving (61) for $\alpha+1$ and $r+s=3$. This completes the proof of Lemma 10 . As in $\S 3$, we only require

$$
\begin{aligned}
& \pi\left(\delta_{r s}^{\alpha}\right) \geqq 2 \alpha-1, \\
& \pi\left(\varepsilon_{r s}^{\alpha}\right) \geqq 2 \alpha .
\end{aligned}
$$

We now choose $K_{2 \alpha-1}$ not divisible by 13 so that

$$
k_{\alpha+1,1} \equiv K_{2 \alpha-1} k_{\alpha 1}\left(\bmod 13^{2 \alpha-1}\right),
$$

which is possible by (56). Then (64) shows that

$$
k_{\alpha+1, r} \equiv K_{2 \alpha-1} k_{\alpha r}\left(\bmod 13^{2 \alpha-1}\right) \quad(r \geqq 1),
$$

so that by $(50)$

$$
L_{2 \alpha+1}(x) \equiv K_{2 \alpha-1} L_{2 \alpha-1}(x)\left(\bmod 13^{2 \alpha-1}\right),
$$

and dividing by $f\left(x^{13}\right)$ (which is legitimate since the power series of $f\left(x^{13}\right)$ has leading coefficient unity) and equating coefficients of like powers of $x$, we obtain

$$
P\left(13^{2 \alpha+1} N\right) \equiv K_{2 \alpha-1} P\left(13^{2 \alpha-1} N\right)\left(\bmod 13^{2 \alpha-1}\right),
$$

for all $n \geqq 1$ and $N=24 n-13$. This is Theorem 2 for odd $\alpha$; the proof for even $\alpha$ is analogous. We can also prove, by a detailed examination of $\delta_{21}^{\alpha}$ and $\varepsilon_{21}^{\alpha}$, that Theorem 2 is best possible.

We now observe that $P\left(13^{4} \cdot 22655\right) \equiv 0\left(\bmod 13^{4}\right)$ and hence

$$
P\left(13^{4+2 m} \cdot 22655\right) \equiv 0\left(\bmod 13^{4}\right) \quad(m \geqq 0),
$$

which proves Theorem 7. Next, since $K_{\alpha} \equiv K_{2}\left(\bmod 13^{2}\right)$ for $\alpha \geqq 2$, and $K_{2}=45$ is a primitive root of $13^{2}$, it follows that $K_{\alpha}$ is a primitive root of $13^{\alpha}$. Also, since $k_{\alpha 1}$ and $l_{\alpha 1}$ are not divisible by 13 , we have $P\left(13^{2 \alpha-1} \cdot 11\right)$ and $P\left(13^{2 \alpha} \cdot 23\right)$ not divisible by 13 . Thus $P\left(13^{2 \alpha-1+2 m} \cdot 11\right)$ for $m \geqq 0$ fills all residue classes prime to 13 $\left(\bmod 13^{2 \alpha-1}\right)$ infinitely often, and similarly $P\left(13^{2 \alpha+2 m} \cdot 23\right)$ fills all residue classes prime to $13\left(\bmod 13^{2 \alpha}\right)$ infinitely often. This proves Theorem 8. 
The use of a "recurrence formula" like Theorem 2 to prove an "infinitely often" property is due to Newman [8]. If we define a "Newman sequence" $t(m)$ by

$$
t(m)=P\left(13^{2 m-1} N_{0}\right) \quad(m \geqq 1),
$$

then Theorem 2 shows that

$$
\begin{aligned}
& \pi(t(m+1))=\pi(t(m)) \quad \text { if } \quad \pi(t(m))<2 m-1, \\
& \pi(t(m+1)) \geqq 2 m-1 \quad \text { if } \quad \pi(t(m)) \geqq 2 m-1 .
\end{aligned}
$$

It follows that either

$$
\pi(t(m)) \geqq 2 m-1 \quad \text { for all } m
$$

or

$$
\pi(t(m))=\pi\left(t\left(m_{0}\right)\right)<2 m_{0}-1 \quad \text { for all } m \geqq m_{0} \text {, and some } m_{0} .
$$

The latter seems more likely.

5. Proof of Conjecture 2 for $\alpha=1$ and $\alpha=2$. We define

$$
\begin{aligned}
P_{11}(N) & =p_{11}(n) \text { if } N=24 n+11, n \geqq 0, \\
& =0 \text { otherwise, }
\end{aligned}
$$

and

$$
\begin{aligned}
P_{23}(N) & =p_{23}(n) \text { if } N=24 n+23, n \geqq 0, \\
& =0 \text { otherwise. }
\end{aligned}
$$

We also let $p \geqq 5$ be prime throughout this section. Then Theorem 1 of Newman [9] shows that for all $N$

$$
\begin{gathered}
P_{11}\left(N p^{2}\right)-\left\{k_{11}(p)-(-3 N / p) p^{4}\right\} P_{11}(N)+p^{9} P_{11}\left(N / p^{2}\right)=0, \\
P_{23}\left(N p^{2}\right)-\left\{k_{23}(p)-(-3 N / p) p^{10}\right\} P_{23}(N)+p^{21} P_{23}\left(N / p^{2}\right)=0,
\end{gathered}
$$

where $k_{11}(p)$ and $k_{23}(p)$ are constants.

Next, by computation from the definitions (46) we find

so that

$$
\begin{aligned}
& L_{1}(x) \equiv 11 g(x)(\bmod 13), \\
& L_{2}(x) \equiv 36 g(x)+78 g^{2}(x)\left(\bmod 13^{2}\right),
\end{aligned}
$$

$$
\sum_{n=1}^{\infty} P(13(24 n-13)) x^{n} \equiv 11 x f\left(x^{13}\right) / f^{2}(x) \equiv 11 \sum_{n=1}^{\infty} p_{11}(n-1) x^{n}(\bmod 13),
$$

whence

$$
P(13 N) \equiv 11 P_{11}(N)(\bmod 13),
$$

and

(71) $\sum_{n=1}^{\infty} P\left(13^{2}(24 n-1)\right) x^{n} \equiv 36 x f^{2}\left(x^{13}\right) / f^{3}(x)+78 x^{2} f^{4}\left(x^{13}\right) / f^{5}(x)\left(\bmod 13^{2}\right)$. 
Now if we write the modular equation (17) as an equation in $1 / t$ and compute the sum of the 11 th powers of the roots we obtain

$$
U \phi^{-11}(x) \equiv 112 g^{-5}(x)+65 g^{-4}(x)\left(\bmod 13^{2}\right),
$$

whence

$$
\begin{aligned}
\sum_{n=1}^{\infty} P_{11}(13(24 n-1)) x^{n} & \equiv 112 x f\left(x^{13}\right) f^{10}(x)+65 x^{2} f^{3}\left(x^{13}\right) f^{8}(x)\left(\bmod 13^{2}\right) \\
& \equiv 112 x f\left(x^{13}\right) f^{10}(x)+65 x^{2} f^{4}\left(x^{13}\right) / f^{5}(x)\left(\bmod 13^{2}\right)
\end{aligned}
$$

But $\left(f\left(x^{13}\right)-f^{13}(x)\right)^{2} \equiv 0\left(\bmod 13^{2}\right)$ so that

$$
f^{23}(x)-2 f\left(x^{13}\right) f^{10}(x)+f^{2}\left(x^{13}\right) / f^{3}(x) \equiv 0\left(\bmod 13^{2}\right) .
$$

Combining (71), (72), and (73) we obtain

$$
\begin{aligned}
\sum_{n=1}^{\infty} P\left(13^{2}(24 n-1)\right) x^{n}+ & 4 \sum_{n=1}^{\infty} P_{11}(13(24 n-1)) x^{n} \\
& \equiv 224 x f^{23}(x)+260 x^{2} f^{2}\left(x^{13}\right) / f^{3}(x) \\
& \equiv 146 x f^{23}(x)=146 \sum_{n=1}^{\infty} P_{23}(24 n-1) x^{n}\left(\bmod 13^{2}\right)
\end{aligned}
$$

whence

$$
P\left(13^{2} N\right) \equiv 165 P_{11}(13 N)+146 P_{23}(N)\left(\bmod 13^{2}\right) .
$$

The coefficients on the RHS of (74) are unique $(\bmod 13)$ but not $\left(\bmod 13^{2}\right)$ since from (72) we have

$$
P_{11}(13 N) \equiv 8 P_{23}(N)(\bmod 13) .
$$

Now Conjecture 2 for $\alpha=1$ follows from (68) and (70), observing that $p^{12} \equiv 1$ $(\bmod 13)$ and $p^{6} \equiv(p / 13)=(13 / p)(\bmod 13)$ if $p \neq 13$. Next, if we multiply $(68)$ with $13 N$ for $N$ and (69) by 165 and 146 respectively, and add, Conjecture 2 for $\alpha=2$ can be seen to follow from

$$
165(13 / p) p^{6} P_{11}(13 N)+146 p^{12} P_{23}(N) \equiv 165 P_{11}(13 N)+146 P_{23}(N)\left(\bmod 13^{2}\right)
$$

and

$$
165 p^{12} P_{11}\left(13 N / p^{2}\right)+146 p^{24} P_{23}\left(N / p^{2}\right) \equiv 165 P_{11}\left(13 N / p^{2}\right)+146 P_{23}\left(N / p^{2}\right)
$$

$\left(\bmod 13^{2}\right)$.

Now (77) is equivalent to

$$
\left(p^{12}-1\right)\left\{165 P_{11}\left(13 N / p^{2}\right)+146\left(p^{12}+1\right) P_{23}\left(N / p^{2}\right)\right\} \equiv 0\left(\bmod 13^{2}\right),
$$

and since $p^{12}-1 \equiv 0(\bmod 13)$ we need only consider the expression in curly brackets $(\bmod 13)$, when it becomes $9 P_{11}\left(13 N / p^{2}\right)+6 P_{23}\left(N / p^{2}\right) \equiv 0(\bmod 13)$ by $(75)$. The proof of (76) is similar, and thus Conjecture 2 is proved for $\alpha=2$. 
We can now establish by the method used to prove Theorem 4 in Newman [9]:

LEMMA 11. If $P\left(13^{2} N_{0}\right) \equiv 0\left(\bmod 13^{2}\right)$ and $N_{0}$ is squarefree, then

$$
P\left(13^{2} n^{2} N_{0}\right) \equiv 0\left(\bmod 13^{2}\right) \text { if }(n, 6)=1 .
$$

The choice $N_{0}=479$ proves Theorem 9 . We were unable to find a single "accident" $\left(\bmod 13^{2}\right)$ for $p \leqq 199$ with $p^{2} k$ in Conjecture 2 congruent to $0, \pm 1\left(\bmod 13^{2}\right)$. Accordingly we chose the first two primes (97 and 103) for which $p^{2} k \equiv \pm 1$ $\left(\bmod 13^{2}\right)$; this makes our explicit linear congruence $(15)$ have $97^{3} \cdot 103^{3} \cdot 13^{2}$ as the multiplier of $n$. Actually $k \equiv 0(\bmod 13)$ for $p=59($ as in Theorem 6$)$ and $p=73$ which would give a linear congruence with the multiplier of $13^{2} n$ equal to $59^{4} \cdot 73^{4}>97^{3} \cdot 103^{3}$. In the course of this work we noticed that for small values of $N$ one has the result:

If any (and hence all) of $P\left(13^{2} N\right), P_{11}(13 N)$, and $P_{23}(N)$ is divisible by 13 , then

$$
P\left(13^{2} N\right) \equiv P_{11}(13 N) \equiv-2 P_{23}(N)\left(\bmod 13^{2}\right) .
$$

This would have been a safe conjecture before the advent of computers, for it is a remarkable fact that there are over 200 successful cases of (78) before it fails for $N=24 \cdot 1530+23$, a point well beyond the range of hand calculation. We offer an explanation in Appendix B.

6. Notes on computation. We obtained the cases $\alpha \leqq 4$ of Theorem 2 in 1963 and communicated our results to Dr. Morris Newman, who asked Dr. Kenneth Kloss of the National Bureau of Standards, Washington, if he could find a suitable zero $\left(\bmod 13^{2}\right)$ of $P\left(13^{2} N\right)$. Kloss obtained several using the direct definition of $p(n)$ on an IBM 7090, at the same time as the second author found the first two, using (71), the letters crossing in the post. Later the second author obtained by hand the first zero of $P\left(13^{3} N\right)\left(\bmod 13^{3}\right)$.

The first author, using the ICT Atlas 1 computer of the Science Research Council at Chilton, computed $P\left(13^{6} N\right), P\left(13^{7} N\right)$, and $c\left(13^{6} n\right)\left(\bmod 13^{6}\right)$ up to $N=24 \cdot 40960-1, N=24 \cdot 40960-13$, and $n=40960$ respectively, using (51), (50), and (35) with explicit coefficients. It was rather disappointing that no better than Theorems 3 and 7 was found $\left(13^{3}\right.$ for $c(n)$ and $13^{4}$ for $\left.p(n)\right)$ in view of the surprisingly early appearance of zeros for $P\left(13^{2} N\right)$ and $P\left(13^{3} N\right)$. However we could not have found Conjectures 1 and 2 without the evidence provided by these runs.

We do not regard the results of this paper which require numerical details beyond the capacity of hand calculation as constituting examples of "machine proof." Indeed programmers, and sometimes machines, make mistakes. In so far as a mathematical proof quotes "known" results, the reader who is not prepared to verify directly the whole of relevant previous knowledge can only be sure that the results are highly probable (since the results in published papers can be incorrect). Now all the quoted calculations in this paper have either been verified 
independently by hand and machine (where possible by different methods), or an independent check has been found assuming Conjectures 1 and 2 . In fact we found that

and

$$
P\left(13^{4} \cdot 22655\right) \equiv P\left(13^{4} \cdot 5^{2} \cdot 22655\right) \equiv 0\left(\bmod 13^{4}\right),
$$

$$
c\left(7 \cdot 13^{3}\right) \equiv 0(\bmod 13), c\left(757 \cdot 13^{3}\right) \equiv 0\left(\bmod 13^{2}\right), c\left(5299 \cdot 13^{3}\right) \equiv 0\left(\bmod 13^{3}\right) .
$$

Thus we claim that Conjectures 1 and 2, by increasing the probability that our calculations are correct, contribute to the proofs of Theorems 3 and 7 .

APPENDix A

Proof of Lemma 5. We consider $g=g(x)$ as a function $g(\tau)$ of $\tau$ where $x \equiv \exp (2 \pi i \tau), \operatorname{Im} \tau>0$. Then $g(\tau)$ is an entire function on $\Gamma_{0}(13)$, and hence (see for example Atkin [5], Lemma 9)

$$
13\{g(-1 / 13 \tau)+13 U g(\tau)\}=g^{-1}(\tau)+13^{2} U g(\tau)
$$

is an entire function on the full modular group $\Gamma(1)$. But $g^{-1}(\tau)$ has a simple pole, residue 1 at $\tau=i_{\infty}$ in the local variable $x$, whence

$$
j(\tau)=g^{-1}(\tau)+13^{2} U g(\tau)+\text { constant. }
$$

Next $g^{-1}(-1 / 13 \tau)+13 U g^{-1}(\tau)$ is an entire function on $\Gamma(1)$, which must be constant since it is regular at $i_{\infty}$, so that $U g^{-1}(\tau)=-g(\tau)+$ constant. Thus

$$
U j(\tau)=-g(\tau)+13^{2} U^{2} g(\tau)+\text { constant }
$$

which implies Lemma 5.

\section{APPENDIX B}

We revert to the use of $p$-rather than $P$-notation, and write (78) in the form

If any (and hence all) of $p\left(13^{2} n+162\right), p_{11}(13 n+12)$, and $p_{23}(n)$ is divisible by 13 , then

$$
p\left(13^{2} n+162\right) \equiv p_{11}(13 n+12) \equiv-2 p_{23}(n)\left(\bmod 13^{2}\right) .
$$

We remind the reader that (79) is false, and write

$$
(a)=\prod_{r=0}^{\infty}\left(1-x^{13 r+a}\right)\left(1-x^{13 r+13-a}\right)
$$

and

$$
\begin{aligned}
& A(x)=\sum_{n=0}^{\infty} \alpha_{n} x^{n}=f^{3}\left(x^{13}\right) \cdot(2)(3)(4)(6) \\
& B(x)=\sum_{n=0}^{\infty} \beta_{n} x^{n}=f^{3}\left(x^{13}\right) \cdot(1)(5)(4)(6) \\
& C(x)=\sum_{n=0}^{\infty} \gamma_{n} x^{n}=x f^{3}\left(x^{13}\right) \cdot(1)(5)(2)(3)
\end{aligned}
$$


Then

Also

$$
\begin{gathered}
A(x)=B(x)+C(x), \\
\alpha_{n}=0 \text { if } n \equiv 1,9,11,12(\bmod 13), \\
\beta_{n}=0 \text { if } n \equiv 2,3,7,8(\bmod 13), \\
\gamma_{n}=0 \text { if } n \equiv 0,4,6,10(\bmod 13) .
\end{gathered}
$$

$$
\begin{aligned}
p_{23}(n) & \equiv 4 \alpha_{n}-3 \beta_{n}(\bmod 13), \\
2 p_{23}(n)+p_{11}(13 n+12) & \equiv 27 \alpha_{n}-82 \beta_{n}\left(\bmod 13^{2}\right) .
\end{aligned}
$$

Suppose now that $p_{23}(n) \equiv 0(\bmod 13)$ and $($ for example) $n \equiv 1(\bmod 13)$. Then $\alpha_{n}=0$ and $\beta_{n} \equiv 0(\bmod 13)$. Now the coefficients $\beta_{n}$ are initially very small numerically, indeed $\left|\beta_{n}\right| \leqq 8$ for $n \leqq 700$, so that $\beta_{n} \equiv 0(\bmod 13)$ implies that $\beta_{n}$ is actually zero in these cases, which now implies (79) by (84) and (74).

This explains the phenomenon when $n \not \equiv 5(\bmod 13)$. If $n \equiv 5(\bmod 13)$ there is an analogous explanation involving $p_{11}(n), p_{23}(13 n+5)$, and different $\alpha_{n}, \beta_{n}, \gamma_{n}$. The only cases where (79) fails for $n \leqq 2100$ are $n=1530,1775,1921$, and 1940 .

We have given no proofs in this Appendix B, but in fact can prove everything but (83) quite easily. We know in principle how to give a very long proof of (83), but confess that we have not gone through the details.

\section{APPENDIX C}

The modular equation (17) is

$$
t^{13}+\sum_{r=1}^{13} \sum_{\sigma=[(r+2) / 2]}^{7} \beta_{r \sigma} g^{\sigma} t^{13-r}=0,
$$

and we now give a table of the explicit values of $\beta_{r \sigma}$.

$$
\begin{array}{rrrrrrrr}
\sigma= & 1 & 2 & 3 & 4 & 5 & 6 & \multicolumn{1}{c}{7} \\
r=1 & -11.13 & -36.13^{2} & -38.13^{3} & -20.13^{4} & -6.13^{5} & -13^{6} & -13^{6} \\
2 & & 204.13 & 346.13^{2} & 222.13^{3} & 74.13^{4} & 13^{6 *} & 13^{6} \\
3 & & -36.13 & -\mathbf{1 2 6 . 1 3}^{2} & -\mathbf{1 0 2 . 1 3}^{3} & -\mathbf{3 8 . 1 3}^{4} & -\mathbf{7 . 1 3}^{5} & -7.13^{5} \\
4 & & & 346.13 & \mathbf{4 2 2 . 1 3}^{2} & \mathbf{1 8 4 . 1 3}^{3} & \mathbf{3 7 . 1 3}^{4} & \mathbf{3 . 1 3}^{5} \\
5 & & & -38.13 & -\mathbf{1 0 2 . 1 3}^{2} & -\mathbf{5 6 . 1 3}^{3} & -\mathbf{1 3}^{5 *} & -\mathbf{1 5 . 1 3}^{4} \\
6 & & & & 222.13 & \mathbf{1 8 4 . 1 3}^{2} & \mathbf{5 1 . 1 3}^{3} & \mathbf{5 . 1 3}^{4} \\
7 & & & -20.13 & -\mathbf{3 8 . 1 3}^{2} & -\mathbf{1 3}^{4 *} & -\mathbf{1 9 . 1 3}^{3} \\
8 & & & & 74.13 & \mathbf{3 7 . 1 3}^{2} & \mathbf{5 . 1 3}^{3} \\
9 & & & & -6.13 & -\mathbf{7 . 1 3}^{2} & -\mathbf{1 5 . 1 3}^{2} \\
10 & & & & & 13^{2 *} & \mathbf{3 . 1 3}^{2} \\
11 & & & & & -13 & -7.13 \\
12 & & & & & & 13 \\
13 & & & & & & -1
\end{array}
$$


It is shown in [1] that

$$
13^{r-\sigma} \beta_{r \sigma}=\beta_{2 \sigma-r, \sigma}
$$

so that the symmetry of the columns is provable a priori. Also the inequality

$$
\pi\left(\beta_{r \sigma}\right) \geqq[(13 \sigma-7 r+13) / 14]
$$

of Lemma 1 in fact holds with equality except in the cases marked with an asterisk in the table. It is an open question whether Lemma 1 can be proved a priori without calculation; the only method we can find is to use (85), but this is not sufficient to establish Lemma 1 in the cases boldfaced in the table.

\section{REFERENCES}

1. J. N. O'Brien, Some properties of partitions with special reference to primes other than 5 , 7, and 11, Ph.D. Thesis, Univ. of Durham, England, 1966.

2. G. N. Watson, Ramanujans Vermutung über Zerfällungsanzahlen, J. Reine Angew. Math. 179 (1938), 97-128.

3. J. Lehner, Divisibility properties of the Fourier coefficients of the modular invariant $j(\tau)$, Amer. J. Math. 71 (1949), 136-148.

4. - Further congruence properties of the Fourier coefficients of the modular invariant j( $\tau$ ), Amer. J. Math. 71 (1949), 373-386.

5. A. O. L. Atkin, Proof of a conjecture of Ramanujan, Glasgow Math. J. (to appear).

6. M. Newman, Periodicity modulo $m$ and divisibility properties of the partition function, Trans. Amer. Math. Soc. 97 (1960), 225-236.

7. - Congruences for the coefficients of modular forms and for the coefficients of $j(\tau)$, Proc. Amer. Math. Soc. 9 (1958), 609-612.

8. - Congruences for the coefficients of modular forms and some new congruences for the partition function, Canad. J. Math. 9 (1957), 549-552.

9. - Further identities and congruences for the coefficients of modular forms, Canad. J. Math. 10 (1958), 577-586.

10. S. Ramanujan, On certain arithmetical functions, Trans. Cambridge Philos. Soc. 22 (1916), 159-184.

11. L. J. Mordell, On Mr. Ramanujan's empirical expansions of modular functions, Proc. Cambridge Philos. Soc. 19 (1919), 117-124.

The Atlas Computer laboratory, Chilton, Didcot, England

THE UNIVERSITY, EXETER, ENGLAND 